\title{
Qualidade pós-colheita de banana 'Maçã' tratada com ácido giberélico avaliada por redes neurais artificiais
}

\author{
César Fernandes Aquino(1), Luiz Carlos Chamhum Salomão(1) e Alcinei Místico Azevedo(2)
}

\begin{abstract}
(1)Universidade Federal de Viçosa, Departamento de Fitotecnia, Setor de Fruticultura, Avenida P.H. Rolfs, s/no, Campus Universitário, CEP 36570-900 Viçosa, MG, Brasil. E-mail: cesarfernandesaquino@yahoo.com.br, Isalomao@ufv.br (2)Universidade Federal de Minas Gerais, Instituto de Ciências Agrárias, Avenida Universitária, o 1.000, Bairro Universitário, CEP 39404-547 Montes Claros, MG, Brasil. E-mail: alcineimistico@hotmail.com
\end{abstract}

Resumo - O objetivo deste trabalho foi avaliar o efeito de doses de ácido giberélico $\left(\mathrm{GA}_{3}\right)$ sobre a extensão da vida pós-colheita em banana 'Maçã' e a aplicabilidade de uso de redes neurais artificiais. Utilizou-se o delineamento inteiramente casualizado, em arranjo de parcelas subdivididas, com cinco doses de $\mathrm{GA}_{3}$, nas parcelas, e tempos de avaliação, nas subparcelas, e quatro repetições, com um buquê de três frutos por unidade amostral. Os frutos, colhidos com a casca totalmente verde, foram imersos por $10 \mathrm{~min}$, à temperatura ambiente,

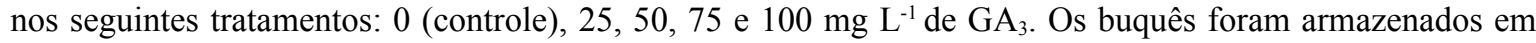
câmara de refrigeração $\left(20 \pm 1^{\circ} \mathrm{C}\right.$, com $91 \%$ de umidade relativa do ar). As redes neurais artificiais são eficientes, proporcionando estimativas do coeficiente de determinação maiores às obtidas por modelos de regressão linear múltipla. Após 19 dias de armazenamento, a dose de $100 \mathrm{mg} \mathrm{L}^{-1}$ de $\mathrm{GA}_{3}$ é mais favorável à conservação pós-colheita de banana 'Maçã', em todas as características avaliadas ao longo do armazenamento.

Termos para indexação: Musa, inteligência computacional, qualidade do fruto, regulador de crescimento.

\section{Gibberellic acid on postharvest quality of banana 'Maçã' evaluated by artificial neural networks}

\begin{abstract}
The objective of this work was to evaluate the effect of gibberellic acid doses on the extent of banana 'Maçã' postharvest life and the applicability of the use of artificial neural networks. A completely randomized design was carried out in a split-plot arrangement, with five doses of $\mathrm{GA}_{3}$ in the plots and evaluation times in the subplots, with four replicates, with a three-fruit bunch by sample unit. Fruit were harvested with totally green peel and were immersed for $10 \mathrm{~min}$, at room temperature, in the following treatments: 0 (control), 25, 50, 75 , and $100 \mathrm{mg} \mathrm{L}^{-1} \mathrm{GA}_{3}$. The bunches were stored in a cold chamber $\left(20 \pm 1^{\circ} \mathrm{C}\right.$, with $91 \%$ air relative humidity). Artificial neural networks are effective, providing estimates of the coefficient of determination higher than those obtained by multiple linear regression models. After 19 days of storage, the dose of $100 \mathrm{mg} \mathrm{L}^{-1} \mathrm{GA}_{3}$ is more favorable to banana 'Maçã' postharvest conservation, in all the evaluated traits during storage.
\end{abstract}

Index terms: Musa, computational intelligence, fruit quality, growth regulator.

\section{Introdução}

OBrasilé o quinto maior produtor mundial de banana, com 6,9 milhões de toneladas em 2013; no entanto, a exportação brasileira foi de apenas 99.216 toneladas naquele ano (Food..., 2015). Além dos problemas inerentes à produção e ao elevado consumo interno, o maior fator restritivo à exportação é a baixa tecnologia de manejo pós-colheita dos frutos (Lichtemberg \& Lichtemberg, 2011). Assim, estratégias para a melhoria da conservação pós-colheita da banana devem ser obtidas.
A banana é colhida antes da completa maturação, por apresentar padrão respiratório climatérico; no entanto, após a colheita, seu amadurecimento é rápido e irreversível, acompanhado de mudanças significativas nos atributos físicos e químicos - como conversão do amido em açúcar, amaciamento da polpa, aroma, sabor - e pigmentação amarela da casca (Mohapatra et al., 2010; Alkarkhi et al., 2011). Isso faz com que a banana madura tenha vida de prateleira relativamente curta, cerca de 6 a 8 dias em temperatura ambiente (Huang et al., 2014). Uma forma de contornar este problema é o uso da cadeia de frio. Entretanto, em armazenamento 
a temperaturas inferiores a $13^{\circ} \mathrm{C}$, os efeitos benéficos à banana são limitados, pois há o desenvolvimento de distúrbios associados ao dano pelo frio, denominado chilling, que causa o escurecimento da casca e a dificuldade de amadurecimento (Nguyen et al., 2003). Esse fato, além de prejudicar a comercialização no mercado interno, contribui para inviabilizar a exportação e a competitividade no mercado externo.

Além do uso do frio, outra alternativa para prolongar a vida pós-colheita dos frutos é a utilização de reguladores de crescimento vegetal, especialmente as giberelinas. $\mathrm{O}$ ácido giberélico $\left(\mathrm{GA}_{3}\right)$ pode ser usado para atrasar o amadurecimento - com o objetivo de manter a qualidade e estender a vida pós-colheita do fruto-, ao reduzir a velocidade de evolução da coloração dos frutos do verde para o amarelo, por impedir a ação das clorofilases e inibir a produção do etileno. Além disso, o uso do $\mathrm{GA}_{3}$ retarda o amolecimento da polpa e o acúmulo de carotenoides, o que evita perdas excessivas na comercialização (Modesto et al., 2006).

$\mathrm{Na}$ literatura científica, estão disponíveis alguns trabalhos sobre o uso do $\mathrm{GA}_{3}$ em diversos frutos, tanto em pré- quanto em pós-colheita, que apresentam resultados satisfatórios quanto à manutenção ou melhoria da qualidade pós-colheita e quanto ao aumento do tempo de vida do fruto em prateleira (Dagar et al., 2012; Weksler et al., 2012; Huang et al., 2014). Em estudo sobre a aplicação do ácido em pré-colheita, Weksler et al. (2012) verificaram que a dose de $50 \mathrm{mg} \mathrm{L}^{-1}$ de $\mathrm{GA}_{3}$, em pêssego e nectarina, foi responsável pela maior firmeza dos frutos. Da mesma forma, Dagar et al. (2012) concluíram que a aplicação pré-colheita de $60 \mathrm{mg} \mathrm{L}^{-1}$ de $\mathrm{GA}_{3}$ se traduz em maior tempo de conservação do pêssego a frio. Huang et al. (2014) avaliaram o efeito do $\mathrm{GA}_{3}$, em pós-colheita da banana (AAA), e constataram que os frutos tratados com $50 \mathrm{mg} \mathrm{L}^{-1}$ de $\mathrm{GA}_{3}$, combinado com $10 \mathrm{mg} \mathrm{L}^{-1}$ de fenilureia, mantiveram-se firmes após 16 dias de armazenamento a $23^{\circ} \mathrm{C}$ e $75-90 \%$ de umidade relativa. Além disso, esse tratamento atrasou o aparecimento do pico respiratório, a mudança da cor da casca e a tranformação do amido em açúcares solúveis.

Pesquisas com o uso do $\mathrm{GA}_{3}$ na pós-colheita de banana são importantes, sobretudo, por ser este um fruto bastante perecível e com tempo de vida em prateleira relativamente curto. Isso favoreceria o transporte para o mercado interno e externo, principalmente para cultivares que, por falta de adequada tecnologia pós-colheita, não suportam certas condições de armazenamento e transporte a longas distâncias. No entanto, são escassas na literatura científica pesquisas que avaliem o efeito de $\mathrm{GA}_{3}$ em pós-colheita de banana 'Maçã'. Portanto, é necessário identificar o efeito deste hormônio e as dosagens favoráveis à melhor conservação pós-colheita dessa fruta.

Para a seleção das melhores doses de produtos em estudos pós-colheita, geralmente, são utilizados modelos de regressão linear múltipla, cujos parâmetros são estimados pelos métodos dos quadrados mínimos ordinários (Lattin et al., 2011). Porém, nem sempre estes modelos possibilitam um bom ajuste (Ghorbani et al., 2016; Khademi \& Behfarnia, 2016; Stojanovic et al., 2016) e, consequentemente, o estudo de características da qualidade pós-colheita em razão de doses de $\mathrm{GA}_{3}$ e tempo de armazenamento da banana pode não ser fidedigna. Isto pode comprometer as conclusões sobre o efeito do $\mathrm{GA}_{3}$ e das dosagens que proporcionam melhor qualidade pós-colheita. Nesta situação, as redes neurais artificiais tornam-se bastante vantajosas, pois são uma alternativa como aproximadores universais de funções complexas, são não paramétricas, não necessitam de informações detalhadas sobre o sistema a ser modelado, toleram perda de dados e possibilitam o ajuste de fenômenos de natureza linear e não linear (Azevedo et al., 2015).

O objetivo deste trabalho foi avaliar o efeito de doses de ácido giberélico $\left(\mathrm{GA}_{3}\right)$ sobre a extensão da vida pós-colheita de banana 'Maçã' e a aplicabilidade de uso de redes neurais artificiais.

\section{Material e Métodos}

Cachos de bananeira 'Maçã' (Musa sp. AAB) foram colhidos de plantas espaçadas em 3,5x2,5 m, em pomar experimental com sete anos de idade, na Universidade Federal de Viçosa, em Viçosa, MG, a 2045'S e $42^{\circ} 52^{\prime} \mathrm{W}$, à altitude de $648 \mathrm{~m}$. Os cachos foram colhidos na fase pré-climatérica, no estádio de cor 1 (casca totalmente verde); deles, selecionaram-se a segunda, terceira e quarta pencas, das quais se eliminaram os frutos danificados, doentes e malformados.

Após a colheita, as pencas foram decompostas em buquês com 3 frutos cada uma, que foram lavados com água e detergente neutro a $0,2 \%$, por $5 \mathrm{~min}$, para coagulação do látex e limpeza superficial. Posteriormente, os buquês foram secos ao ar até que 
não se observassem mais gotas de água na superfície dos frutos. Os frutos que compuseram cada buquê foram selecionados pela uniformidade quanto ao peso e dimensões. Posteriormente, foram imersos em solução fungicida procloraz $\left(0,22 \mathrm{~g} \mathrm{~L}^{-1}\right)$ por $5 \mathrm{~min}$ e secos ao ar.

Após a secagem, os buquês foram divididos aleatoriamente em 5 grupos, para receber os tratamentos $(\mathrm{T})$, que consistiram das seguintes concentrações de $\mathrm{GA}_{3}$ (Sigma-Aldrich, St. Louis, MO, EUA): T1, apenas água (controle); T2, $25 \mathrm{mg}$ $\mathrm{L}^{-1}$; T3, $50 \mathrm{mg} \mathrm{L}^{-1}$; T4, $75 \mathrm{mg} \mathrm{L}^{-1}$; e T5, $100 \mathrm{mg} \mathrm{L}^{-1}$. Os buquês foram imersos nas soluções por $10 \mathrm{~min}$, à temperatura ambiente. Em seguida, os buquês foram completamente secos ao ar, acondicionados em caixas de plástico e armazenados em câmara de refrigeração, a $20 \pm 1^{\circ} \mathrm{C}$, com $91 \%$ de umidade relativa do ar, até o completo amadurecimento.

As avaliações foram feitas no dia do armazenamento (dia 0) e, posteriormente, aos dias 2, 4, 6, 8, 10, 13, 16 e 19 de armazenamento. Foram retirados quatro buquês por tratamento, para a avaliação da perda de massa de matéria fresca, firmeza da polpa e parâmetros de cor da casca. Em seguida, estes frutos foram descascados, processados em liquidificador doméstico, e uma amostra composta da polpa foi retirada para a análise de sólidos solúveis e acidez titulável. Amostras da casca também foram retiradas, para a determinação do teor de clorofila.

Para determinar a perda de massa de matéria fresca, utilizou-se uma balança semianalítica, e os resultados foram expressos em percentagem. A firmeza da polpa foi aferida por penetrômetro Shimpo DFS 100 (Digital Force Gauge, Cedarhurst, NY, EUA), com ponteira de $8 \mathrm{~mm}$ de diâmetro, na região mediana de cada fruto, após a retirada de uma porção da casca; os resultados foram expressos em newtons. Utilizou-se o colorímetro modelo CR 10 (Konica-Minolta Inc., Tokyo, Japan), para fornecimento dos valores do ângulo hue da casca.

$\mathrm{O}$ teor de sólidos solúveis foi determinado com o auxílio de um refratômetro digital portátil Atago N1. Para a acidez titulável, amostras compostas de 5,0 g cada uma foram tituladas com solução de $\mathrm{NaOH} 0,05$ $\mathrm{N}$, previamente padronizada com biftalato de potássio. O teor de clorofila foi determinado em amostras de $2 \mathrm{~g}$ de casca, tendo-se utilizado acetona a $80 \%$ para a extração dos pigmentos, conforme Smith \& Benitez (1955).
Para a avaliação da significância dos efeitos das fontes de variação pelo teste $\mathrm{F}$, utilizou-se um delineamento inteiramente casualizado, em arranjo de parcelas subdivididas. Assim, foram alocadas nas parcelas cinco doses de $\mathrm{GA}_{3}$ e, nas subparcelas, os dias de avaliação, com quatro repetições (buquês) de três frutos. Todas as análises estatísticas foram feitas pelo programa R (R Core Team, 2013). Para verificar a eficiência dos efeitos das fontes de variação pelo teste F, utilizou-se a função aov do pacote Stats.

Para o ajuste dos caracteres qualitativos dos frutos, em função das doses de $\mathrm{GA}_{3}$ e dias de avaliação, testaram-se 14 modelos de regressão linear múltipla (Tabela 1), cujo ajuste foi feito com o método dos quadrados mínimos e com o auxílio da função $1 \mathrm{~m}$ do pacote Stats. Testou-se também o modelo de redes neurais artificiais do tipo percéptron multicamadas (MLP, multi-layer perceptron). Para verificar a qualidade do ajuste dos modelos, recorreu-se ao coeficiente de determinação, com base na validação cruzada. Para isso, as 180 observações, para cada uma das características -5 doses $\times 9$ dias de avaliação $\times 4$ repetições -, foram divididas aleatoriamente em duas amostras. A primeira amostra, destinada à calibração do modelo, foi composta por $75 \%$ das observações (135 observações). A segunda amostra, utilizada para verificar a eficiência do ajuste do modelo, foi composta por $25 \%$ dos dados (45 observações).

Tabela 1. Modelos de regressão múltipla testados para descrever caracteres qualitativos $\left(\mathrm{z}_{\mathrm{i}}\right)$ em frutos de bananeira 'Maçã', em função de doses de $\mathrm{GA}_{3}\left(\mathrm{x}_{\mathrm{i}}\right)$ e dias pós-colheita $\left(\mathrm{y}_{\mathrm{i}}\right)$.

\begin{tabular}{|c|c|}
\hline Modelo & Função \\
\hline 1 & $z_{i}=a+b x_{i}+e_{i}$ \\
\hline 2 & $z_{i}=a+b y_{i}+e_{i}$ \\
\hline 3 & $z_{i}=a+b x_{i}+c y_{i}+e_{i}$ \\
\hline 4 & $z_{i}=a+b x_{i}+c x_{i}^{2}+d y_{i}+e_{i}$ \\
\hline 5 & $z_{i}=a+b x_{i}+c y_{i}+d y_{i}^{2}+e_{i}$ \\
\hline 6 & $z_{i}=a+b x_{i}+c x_{i}^{2}+d y_{i}+f y_{i}^{2}+e_{i}$ \\
\hline 7 & $z_{i}=a+b x_{i}+c y_{i}+d x_{i} y_{i}+e_{i}$ \\
\hline 8 & $z_{i}=a+b x_{i}+c x_{i}^{2}+d y_{i}+f x_{i} y_{i}+e_{i}$ \\
\hline 9 & $z_{i}=a+b x_{i}+c y_{i}+d y_{i}^{2}+f x_{i} y_{i}+e_{i}$ \\
\hline 10 & $\mathrm{z}_{\mathrm{i}}=\mathrm{a}+\mathrm{bx}_{\mathrm{i}}+\mathrm{cx}_{\mathrm{i}}^{2}+\mathrm{dy}_{\mathrm{i}}+\mathrm{fy}_{\mathrm{i}}^{2}+\mathrm{gx}_{\mathrm{i}} \mathrm{y}_{\mathrm{i}}+\mathrm{e}_{\mathrm{i}}$ \\
\hline 11 & $z_{i}=a+b x_{i}+c_{i}^{2}+d y_{i}+f y_{i}^{2}+g x_{i} y_{i}+h x_{i}^{2} y_{i}+e_{i}$ \\
\hline 12 & $z_{i}=a+b x_{i}+c x_{i}^{2}+d y_{i}+f y_{i}^{2}+g x_{i} y_{i}+h x_{i} y_{i}^{2}+e_{i}$ \\
\hline 13 & $z_{i}=a+b x_{i}+c x_{i}^{2}+d y_{i}+f y_{i}^{2}+g x_{i} y_{i}+h x_{i}^{2} y_{i}+j x_{i} y_{i}^{2}+e_{i}$ \\
\hline 14 & $z_{i}=a+b x_{i}+c x_{i}^{2}+d y_{i}+f y_{i}^{2}+g x_{i} y_{i}+h x_{i}^{2} y_{i}+j x_{i}$ \\
\hline
\end{tabular}


Para a modelagem por redes neurais artificiais, os dados foram normalizados para o intervalo entre 0 e 1, pela função normalizeData do pacote RSNNS (Bergmeir \& Benítez, 2012). Para o desenvolvimento das redes do tipo MLP, utilizou-se a função mlp do pacote RSNNS, com algoritmo backpropagation e taxa de aprendizado de 0,1 . O número máximo de épocas de treinamento foi arbitrado como 500. A função de ativação para a camada intermediária foi a logística e, para a camada de saída, foi a linear. A arquitetura de rede utilizada foi arbitrada com uma única camada intermediária com cinco neurônios. A camada de entrada foi composta pela dose de $\mathrm{GA}_{3} \mathrm{e}$ dia de avaliação de cada amostra. Na camada de saída, considerou-se a característica a ser predita (indicadores de qualidade pós-colheita). A topologia desta rede é apresentada na Figura 1. Tendo-se em vista que, no início do treinamento, os parâmetros livres são gerados aleatoriamente, e que esses valores iniciais podem influenciar o resultado final do treinamento (Soares et al., 2014), cada configuração de RNA foi treinada 100 vezes. A seleção da rede que proporcionou o melhor ajuste foi feita considerando-se o menor erro quadrático médio (EQM) para a amostra de validação. Para a identificação das variáveis independentes mais

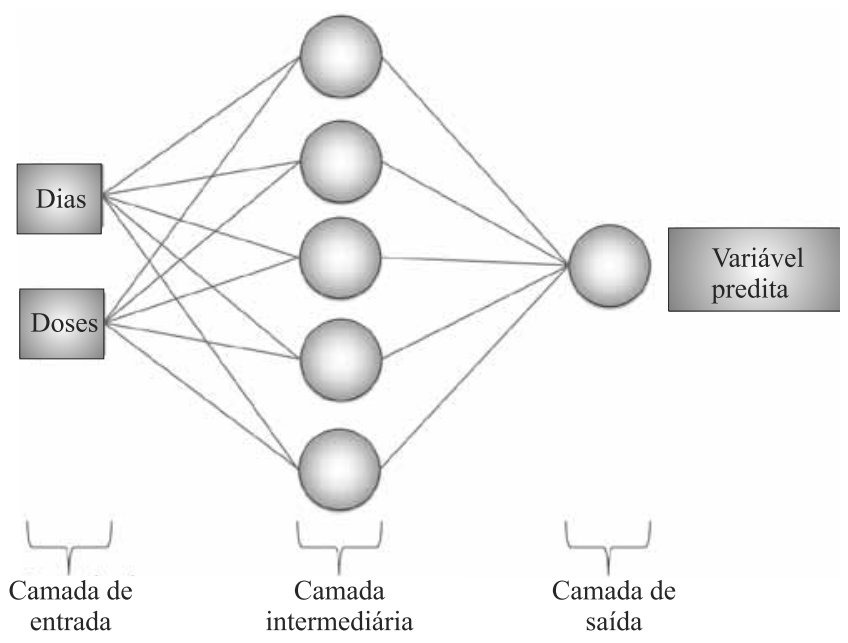

Figura 1. Arquitetura de redes do tipo percéptron multicamadas, utilizadas na predição de caracteres qualitativos de banana 'Maçã' - perda de massa de matéria fresca, ângulo hue, teor de clorofila, firmeza da polpa, sólidos solúveis e acidez titulável -, em função de doses de $\mathrm{GA}_{3}\left(0,25,50,75\right.$ e $\left.100 \mathrm{mg} \mathrm{L}^{-1}\right)$ e dias de avaliação $(0,2,4$, $6,8,10,13,16$ e 19). importantes no estudo das redes neurais, obtevese a importância relativa pelo método de Garson (1991), recorrendo-se à função garson do pacote NeuralNetTools.

Como o ajuste obtido por RNAs foi melhor do que os encontrados pelas regressões múltiplas, obtiveramse valores preditos das características estudadas pela função predict do pacote RSNNS. A partir dos valores preditos, obtiveram-se os gráficos de superfície de resposta, com auxílio do programa Sigma-Plot 11.0.

\section{Resultados e Discussão}

Observou-se pelo teste $\mathrm{F}$, a $5 \%$ de probabilidade, que as doses de $\mathrm{GA}_{3}$ tiveram efeito significativo sobre a perda de massa de matéria fresca, sólidos solúveis e acidez titulável (Tabela 2). Ademais, para o efeito dos dias de avaliação e da interação entre as doses e dias de avaliação, encontraram-se efeitos significativos $(\mathrm{p}<0,05)$ em todas as características.

Para os coeficientes de determinação $\left(R^{2}\right)$ obtidos, verificaram-se maiores estimativas para as características perda de massa de matéria fresca, acidez titulável e teor de clorofila (Tabela 3). As menores estimativas de $\mathrm{R}^{2}$ foram encontradas para o ângulo hue e a firmeza de polpa. Isto indica que as variações encontradas nestas características não são bem explicadas pelas variações das doses de $\mathrm{GA}_{3}$ e dos dias de avaliação, em comparação às demais características avaliadas. As maiores estimativas de $\mathrm{R}^{2}$ na amostra de calibração, em comparação à amostra de validação, foram encontradas na maioria dos casos. Recomenda-se o uso de duas amostras - uma para o ajuste de modelos (amostra de calibração) e outra para a validação (método de validação cruzada) -, pois isto evita a seleção de modelos com ajuste exagerado, o que compromete a validade externa do modelo de regressão (Lattin et al., 2011).

Para todos os casos estudados, os coeficientes de determinação obtidos pelas redes neurais artificiais (MLP) apresentaram maiores estimativas do que os obtidos por regressão múltipla (Tabela 3), tanto na amostra de calibração quanto na amostra de validação. Isto pode ser justificado pela grande eficiência das redes neurais na identificação de padrões entre as variáveis dependentes e independentes, o que leva à obtenção de ajustes com melhor qualidade (Azevedo et al., 2015). Os melhores ajustes das redes neurais, 
em comparação aos modelos de regressão múltipla, também foram verificados por Ghorbani et al. (2016), Stojanovic et al. (2016) e Khademi \& Behfarnia (2016). A variável independente que apresentou maior contribuição ao estudo por redes neurais, de acordo com o método Garson (1991), foi o número de dias de avaliação (Tabela 4). Isto indica que os parâmetros de qualidade pós-colheita foram mais influenciados pelo

Tabela 2. Estimativas do valor p, obtidos pelo teste $\mathrm{F}$ das fontes de variação controladas no estudo de caracteres de qualidade de banana 'Maçã', em função de doses de $\mathrm{GA}_{3}$ e dias de avaliação.

\begin{tabular}{|c|c|c|c|c|c|c|}
\hline Fonte de variação & $\begin{array}{l}\text { Perda de massa de } \\
\text { matéria fresca }\end{array}$ & Ângulo hue & Firmeza da polpa & Sólidos solúveis & Acidez titulável & Teor de clorofila \\
\hline Doses & 0,01 & 0,13 & 0,86 & $<0,01$ & $<0,01$ & 0,13 \\
\hline Dias & $<0,01$ & $<0,01$ & $<0,01$ & $<0,01$ & $<0,01$ & $<0,01$ \\
\hline Doses $\mathrm{x}$ dias & $<0,01$ & $<0,01$ & $<0,01$ & $<0,01$ & $<0,01$ & 0,02 \\
\hline $\mathrm{CV}_{1}(\%)^{(1)}$ & 42,05 & 2,47 & 18,70 & 9,79 & 8,81 & 7,34 \\
\hline $\mathrm{CV}_{2}(\%)^{(2)}$ & 15,76 & 4,06 & 18,21 & 12,29 & 8,62 & 7,02 \\
\hline
\end{tabular}

${ }^{(1)} \mathrm{CV}_{1}$, coeficiente de variação residual associado aos efeitos das doses de $\mathrm{GA}_{3} \cdot{ }^{(2)} \mathrm{CV}_{2}$, coeficiente de variação residual associado aos efeitos dos dias de avaliação.

Tabela 3. Coeficientes de determinação $\left(\mathrm{R}^{2}\right)$ de 14 modelos regressão múltipla e do modelo de redes neurais artificiais, do tipo percéptron multicamadas (MLP), obtidos para a amostra de calibração e amostra de validação, na modelagem de caracteres qualitativos de banana 'Maçã', em função de doses de $\mathrm{GA}_{3}$ e dias de avaliação.

\begin{tabular}{|c|c|c|c|c|c|c|}
\hline Modelo & $\begin{array}{c}\text { Perda de massa de } \\
\text { matéria fresca }\end{array}$ & Ângulo hue & Firmeza da polpa & Sólidos solúveis & Acidez titulável & Teor de clorofila \\
\hline & \multicolumn{6}{|c|}{$\mathrm{R}^{2}$ para a amostra de calibração } \\
\hline 1 & 0,001 & 0,001 & 0,001 & 0,000 & 0,003 & 0,005 \\
\hline 2 & 0,893 & 0,741 & 0,739 & 0,837 & 0,777 & 0,936 \\
\hline 3 & 0,896 & 0,741 & 0,739 & 0,838 & 0,782 & 0,938 \\
\hline 4 & 0,915 & 0,742 & 0,740 & 0,838 & 0,783 & 0,938 \\
\hline 5 & 0,914 & 0,790 & 0,799 & 0,872 & 0,816 & 0,950 \\
\hline 6 & 0,934 & 0,791 & 0,800 & 0,872 & 0,817 & 0,950 \\
\hline 7 & 0,896 & 0,741 & 0,739 & 0,838 & 0,783 & 0,939 \\
\hline 8 & 0,915 & 0,742 & 0,740 & 0,838 & 0,784 & 0,939 \\
\hline 9 & 0,915 & 0,791 & 0,799 & 0,872 & 0,817 & 0,951 \\
\hline 10 & 0,934 & 0,791 & 0,800 & 0,872 & 0,818 & 0,952 \\
\hline 11 & 0,943 & 0,793 & 0,803 & 0,873 & 0,818 & 0,952 \\
\hline 12 & 0,937 & 0,791 & 0,801 & 0,873 & 0,819 & 0,952 \\
\hline 13 & 0,945 & 0,793 & 0,803 & 0,873 & 0,819 & 0,952 \\
\hline 14 & 0,945 & 0,793 & 0,803 & 0,873 & 0,819 & 0,952 \\
\hline \multirow[t]{2}{*}{ MLP } & 0,948 & 0,832 & 0,853 & 0,879 & 0,937 & 0,955 \\
\hline & \multicolumn{6}{|c|}{$\mathrm{R}^{2}$ para a amostra de validação } \\
\hline 1 & 0,032 & 0,000 & 0,01 & 0,000 & 0,014 & 0,035 \\
\hline 2 & 0,856 & 0,677 & 0,661 & 0,676 & 0,697 & 0,934 \\
\hline 3 & 0,857 & 0,680 & 0,661 & 0,669 & 0,691 & 0,928 \\
\hline 4 & 0,892 & 0,673 & 0,648 & 0,664 & 0,686 & 0,929 \\
\hline 5 & 0,883 & 0,805 & 0,747 & 0,749 & 0,797 & 0,951 \\
\hline 6 & 0,918 & 0,799 & 0,733 & 0,745 & 0,791 & 0,951 \\
\hline 7 & 0,857 & 0,680 & 0,661 & 0,669 & 0,695 & 0,927 \\
\hline 8 & 0,892 & 0,673 & 0,648 & 0,664 & 0,689 & 0,928 \\
\hline 9 & 0,884 & 0,804 & 0,747 & 0,750 & 0,801 & 0,951 \\
\hline 10 & 0,919 & 0,799 & 0,733 & 0,745 & 0,796 & 0,952 \\
\hline 11 & 0,933 & 0,784 & 0,723 & 0,749 & 0,793 & 0,951 \\
\hline 12 & 0,913 & 0,801 & 0,730 & 0,744 & 0,794 & 0,952 \\
\hline 13 & 0,932 & 0,787 & 0,717 & 0,748 & 0,791 & 0,951 \\
\hline 14 & 0,932 & 0,787 & 0,717 & 0,748 & 0,791 & 0,951 \\
\hline MLP & 0,942 & 0,822 & 0,824 & 0,888 & 0,953 & 0,952 \\
\hline
\end{tabular}


fator tempo de armazenamento do que pelo fator dose de $\mathrm{GA}_{3}$.

Verificou-se pelo teste pareado de Wilcoxon que, a 5\% de probabilidade, não há evidências suficientes para rejeitar a hipótese nula, na comparação dos valores preditos pelas redes neurais com os observados, para a amostra de calibração e validação (Tabela 5). Neste caso, considera-se como hipótese nula que os valores observados (dados reais) não diferem dos preditos pelas RNAs. Isto indica a eficiência de predição das redes neurais, encontrada no presente trabalho, conforme já relatado anteriormente. Com o melhor ajuste das RNAs, as superfícies de resposta foram obtidas a partir de valores preditos (Figura 2).

A média de perda de massa de matéria fresca total, em todos os tratamentos, foi 3,96\%, após 19 dias de armazenamento. Os frutos do controle $\left(0 \mathrm{mg} \mathrm{L}^{-1}\right.$ de $\left.\mathrm{GA}_{3}\right)$ e os do tratamento com $100 \mathrm{mg} \mathrm{L}^{-1}$ de $\mathrm{GA}_{3}$ apresentaram as maiores perdas de massa de matéria fresca (Figura $2 \mathrm{~A}$ ), que são em média 20\% superiores aos dos outros tratamentos, ao final do período de avaliação. No entanto, a perda de massa de matéria fresca observada no presente trabalho foi mínima e não atingiu 5\%, em termos práticos, não comprometeu a qualidade final dos frutos, aos 19 dias de armazenamento. Ao longo do armazenamento, verificou-se a menor perda de massa de matéria fresca com as doses 25 e $50 \mathrm{mg} \mathrm{L}^{-1}$ de $\mathrm{GA}_{3}$. Archana \& Sivachandiran (2015) verificaram em frutos não tratados com $\mathrm{GA}_{3}$ um maior percentual de perda de massa de matéria fresca, em banana 'Kathali' (Sri Lanka). Finger et al. (1995) observaram que a perda de massa de matéria fresca afeta a fase pré-climatérica de banana 'Robusta' (Musa acuminata Colla). Os mesmos autores relataram que a perda de $5 \%$ de matéria fresca reduz o período pré-climatérico em 7 dias, e a de $10 \%$ o reduz em 10 dias. Entretanto, no presente trabalho, não se observou relação entre a perda de massa de matéria fresca e a antecipação do amadurecimento dos frutos. Isso pode ter ocorrido em razão da perda de massa de matéria fresca inferior a 5\% no presente trabalho.

Quanto ao ângulo hue (Figura $2 \mathrm{~B}$ ), em todos os tratamentos, os frutos permaneceram com a coloração verde até por volta do $8 .^{\circ}$ dia de armazenamento, o que mostra que os frutos ainda não se encontravam em processo de amadurecimento. A partir desse período, com o incremento dos teores de sólidos solúveis, degradação da clorofila, e perda da firmeza, observou-se o início do amadurecimento dos frutos. Segundo Yang et al. (2009), a perda da cor verde é um dos sintomas de amadurecimento que conduz à senescência dos frutos. Aos 16 dias de armazenamento, os frutos tratados com $100 \mathrm{mg} \mathrm{L}^{-1}$ de $\mathrm{GA}_{3}$ apresentaram ângulo hue de 92,82, o que é indicativo de possível ação do $\mathrm{GA}_{3}$ para retardar a degradação da clorofila, uma vez que as giberelinas apresentam a função de retardar o amadurecimento dos frutos, o que afeta principalmente as mudanças de cor da casca (Modesto et al., 2006). No entanto, nos frutos-testemunha o ângulo foi 87,39 , o que indica que, nos frutos não tratados com $\mathrm{GA}_{3}$, a degradação da clorofila foi mais intensa e promoveu a revelação dos carotenoides existentes. Ao final do período de armazenamento, houve estabilização do ângulo hue, com valor médio de 87 , o que indica a coloração

Tabela 4. Contribuição relativa das variáveis explicativas (doses e dias de avaliação), obtida pelo método Garson, para a predição de caracteres qualitativos de banana 'Maçã', em função de doses de $\mathrm{GA}_{3}$ e dias de avaliação por percéptrons multicamadas.

\begin{tabular}{lccccc}
\hline Variáveis & $\begin{array}{c}\text { Perda de massa de } \\
\text { matéria fresca }\end{array}$ & Ângulo hue & Firmeza da polpa & Sólidos solúveis & Acidez titulável \\
\hline Doses & 0,443 & 0,447 & 0,417 & 0,342 & 0,256 \\
Dias & 0,557 & 0,553 & 0,583 & 0,657 & 0,204 \\
\hline
\end{tabular}

Tabela 5. Estimativas do valor p para o teste pareado de Wilcoxon, para a comparação das estimativas de caracteres qualitativos de banana 'Maçã', observados e preditos por redes neurais artificiais, nas amostras de calibração e de validação.

\begin{tabular}{lccccc}
\hline Amostras & $\begin{array}{c}\text { Perda de massa de } \\
\text { matéria fresca }\end{array}$ & Ângulo hue & Firmeza da polpa & Sólidos solúveis & Acidez titulável \\
\hline Calibração & 0,594 & 0,716 & 0,692 & 0,656 & 0,550 \\
Validação & 0,965 & 0,325 & 0,799 & 0,948 & 0,763 \\
\hline
\end{tabular}



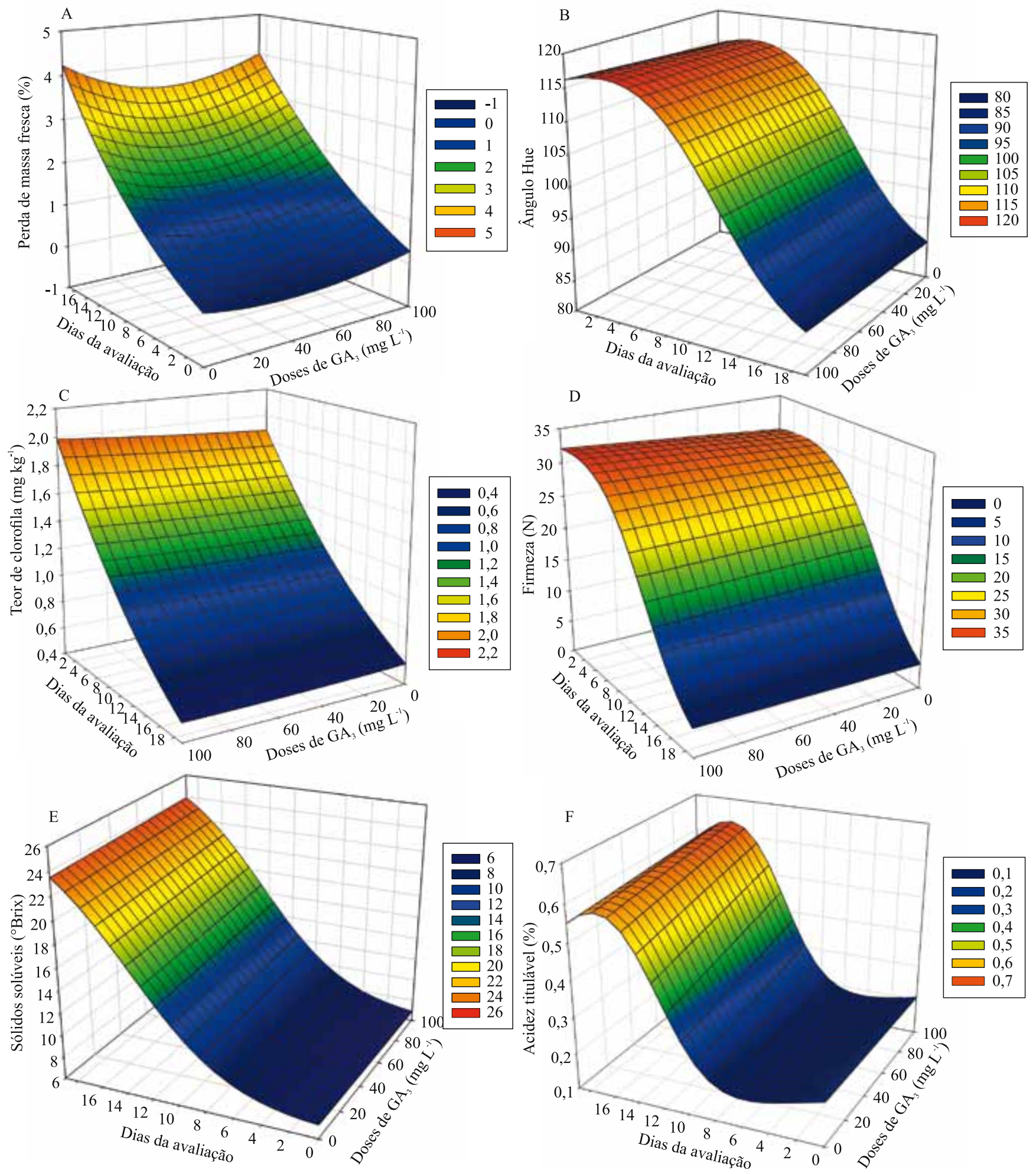

Figura 2. Superfícies de resposta para as características perda de massa de matéria fresca (A), ângulo hue (B), teor de clorofila (C), firmeza de polpa (D), sólidos solúveis (E) e acidez titulável (F), em frutos de bananeira 'Maçã', em função de doses de $\mathrm{GA}_{3}\left(0,25,50,75\right.$ e $\left.100 \mathrm{mg} \mathrm{L}^{-1}\right)$ e dias de avaliação $(0,2,4,6,8,10,13,16$ e 19), obtidas por redes neurais do tipo percéptron multicamadas. 
amarela típica da casca do fruto maduro, que está relacionada com a degradação da clorofila na casca e a exposição dos pigmentos carotenoides. Essa mudança de cor é consequência da ação enzimática na estrutura da clorofila que permite evidenciar os carotenoides (Newilah et al., 2009). Huang et al. (2014) observaram que bananas tratadas com $50 \mathrm{mg} \mathrm{L}^{-1} \mathrm{de}_{\mathrm{GA}_{3}}$, combinado com $10 \mathrm{mg} \mathrm{L}^{-1}$ de fenilureia, retardaram a mudança da cor da casca, em comparação à testemunha, após 16 dias de armazenamento a $23^{\circ} \mathrm{C}$ e $75-90 \%$ de umidade relativa. Rossetto et al. (2004) verificaram que fatias de banana tratadas em solução de manitol $\left(120 \mathrm{mmol} \mathrm{L}^{-1}\right)$, com $0,1 \mathrm{mmol} \mathrm{L}^{-1}$ de $\mathrm{GA}_{3}$, apresentaram atraso no amadurecimento, a cor da casca permaneceu verde por mais tempo, e não ocorreu aroma típico de fruto maduro.

O teor de clorofila reduziu-se com o armazenamento dos frutos, com inclinação na superfície para as maiores doses de $\mathrm{GA}_{3}$, isto é, as maiores doses influenciaram positivamente a manutenção da coloração verde e retardaram a degradação da clorofila na casca, ao longo de todo o período de armazenamento dos frutos (Figura $2 \mathrm{C}$ ). A mudança de cor da casca do estádio de cor 1 (casca totalmente verde) para 6 (casca totalmente amarela) é caracterizada pelo decréscimo dos teores de clorofila, ou seja, no dia zero, a média de todos os tratamentos foi de $22,69 \mathrm{mg} \mathrm{kg}^{-1}$ e se reduziu para o teor médio de 4,13 mg kg-1, após 19 dias de armazenamento, o que evidencia o aparecimento da coloração amarela. Essa mudança de cor é consequência da ação enzimática na estrutura da clorofila e pode ser relacionada ao acúmulo de açúcares solúveis na casca, com o amadurecimento dos frutos (Newilah et al., 2009; Yang et al., 2009). A degradação da clorofila permite evidenciar os carotenoides na casca. Com o aparecimento dos carotenoides na casca, não houve diferenças entre as doses, no restante do período de armazenamento, conforme verificado para o ângulo hue.

A firmeza da polpa sofreu pouca alteração até o 8. ${ }^{\circ}$ dia de armazenamento. A partir daí, em todos os tratamentos, a firmeza sofreu queda brusca até o final do armazenamento, em consequência do amadurecimento dos frutos (Figura 2 D). Nota-se inclinação na superfície para as maiores doses de $\mathrm{GA}_{3}$, isto é, as maiores doses influenciam positivamente a manutenção da firmeza da polpa até o $12 .^{\circ}$ dia de armazenamento (Figura $2 \mathrm{D}$ ). No entanto, a partir daí, não se observa mais a influência das doses de $\mathrm{GA}_{3}$ na manutenção da firmeza dos frutos tratados, em comparação aos frutos da testemunha. Huang et al. (2014) observaram que os frutos tratados com $10 \mathrm{mg} \mathrm{L}^{-1}$ de fenilureia e $50 \mathrm{mg} \mathrm{L}^{-1}$ de $\mathrm{GA}_{3}$ mantiveram-se firmes, após 16 dias de armazenamento a $23^{\circ} \mathrm{C}$ e $75-90 \%$ de umidade relativa; no entanto, os frutos-controle se mostravam impróprios para $o$ consumo. A perda de firmeza da polpa está associada às atividades enzimáticas relacionadas à degradação de componentes pécticos da parede celular e da lamela média e à conversão do amido em açúcares, durante o amadurecimento (Mohapatra et al., 2010). Segundo Rossetto et al. (2004), o $\mathrm{GA}_{3}$ afeta as enzimas que degradam o amido, principalmente as amilases e, como o amido tem função estrutural na polpa da banana, era de se esperar que ocorresse atraso da perda de firmeza da polpa, nos frutos tratados com $\mathrm{GA}_{3}$, em relação à testemunha.

$\mathrm{O}$ teor de sólidos solúveis (SS) elevou-se rapidamente, a partir do $8 .^{\circ}$ dia de armazenamento, com o início do amadurecimento dos frutos em todos os tratamentos (Figura 2 E). Coelho et al. (2011) observaram que a banana 'Maçã' armazenada a $21^{\circ} \mathrm{C}$ apresentou baixo teor de sólidos solúveis até sete dias de armazenamento. No presente trabalho, ao final do período de armazenamento, os teores de SS foram bastante próximos, em todos os tratamentos, com 23,8, $24,1,24,7,24,3$ e $24,3^{\circ}$ Brix, para as doses $0,25,50$,

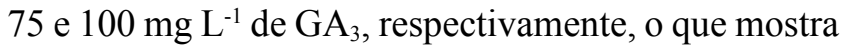
não haver efeito das doses de $\mathrm{GA}_{3}$ quanto ao atraso da conversão do amido em açúcares. Aquino (2014) verificou, em frutos de banana 'Maçã' no estádio de cor 6, o teor de SS de $25,7^{\circ}$ Brix. Os frutos verdes da banana 'Maçã' apresentam cerca de $25 \%$ de amido, que é hidrolisado com o amadurecimento e resulta no acúmulo de açúcares, dos quais 17,28\% são de açúcares solúveis, mais $5,68 \%$ de amido restante, no estádio de cor 6 (Aquino, 2014), e o teor de sólidos solúveis é indicativo do teor de açúcares existente na fruta.

Para a acidez titulável, nota-se o incremento dos teores somente a partir do $8 .^{\circ}$ dia de armazenamento, em frutos de todos os tratamentos, com exceção da testemunha, em que a mudança se iniciou somente a partir do $10 .^{\circ}$ dia de armazenamento, e passou de 0,2 para $0,6 \mathrm{~g}$ de ácido málico por $100 \mathrm{~g}$ de polpa. Do $12 .^{\circ}$ ao $19 .^{\circ}$ dia de armazenamento, observam-se maiores teores de acidez, com o aumento das doses de $\mathrm{GA}_{3}$ (Figura 2 F). Segundo Bleinroth (1990), a banana no estádio verde caracteriza-se por apresentar baixa acidez, 
que aumenta com o decorrer do amadurecimento até atingir o máximo, quando a casca está totalmente amarela, para posteriormente decrescer, o que indica o início da senescência. Esse fenômeno ocorre em razão da solubilização de substâncias pécticas, como consequência da atividade enzimática, com o amadurecimento dos frutos (Prill et al., 2012).

$\mathrm{O}$ tratamento com $\mathrm{GA}_{3}$ pode ser promissor na manutenção e melhoria da qualidade pós-colheita da banana 'Maçã', pois prolonga a vida útil dos frutos. Trabalhos futuros, com aplicação de tempos mais longos de imersão dos frutos em $\mathrm{GA}_{3}$ e maiores doses deste regulador vegetal, devem ser realizados para controlar o amadurecimento dos frutos, pois podem apresentar resultados melhores que os obtidos no presente trabalho.

\section{Conclusões}

1. A dose de $100 \mathrm{mg} \mathrm{L}^{-1}$ de $\mathrm{GA}_{3}$ é mais favorável à conservação pós-colheita da banana 'Maçã', para as características ângulo hue, teor de clorofila, firmeza e sólidos solúveis.

2. As redes neurais artificiais são eficientes, proporcionando estimativas do coeficiente de determinação maiores às obtidas pelos modelos de regressão linear múltipla.

\section{Agradecimentos}

Ao Conselho Nacional de Desenvolvimento Científico e Tecnológico (CNPq), à Coordenação de Aperfeiçoamento de Pessoal de Nível Superior (Capes) e à Fundação de Apoio à Pesquisa do Estado de Minas Gerais (Fapemig), pela concessão de bolsas de estudos e de pesquisa.

\section{Referências}

ALKARKHI, A.F.M.; RAMLI, S. bin.; YONG, Y.S.; EASA, A.M. Comparing physicochemical properties of banana pulp and peel flours prepared from green and ripe fruits. Food Chemistry, v.129, p.312-318, 2011. DOI: 10.1016/j.foodchem.2011.04.060.

AQUINO, C.F. Características físicas e químicas e potencial antioxidante dos frutos de 15 cultivares de bananeiras. 2014. 131p. Tese (Doutorado) - Universidade Federal de Viçosa, Viçosa.

ARCHANA, U.; SIVACHANDIRAN, S. Effect of application of gibberellic acid $\left(\mathrm{GA}_{3}\right)$ on shelf-life of banana. International Journal of Research in Agriculture and Food Sciences, v.3, p.1-4, 2015
AZEVEDO, A.M.; ANDRADE JÚNIOR, V.C. de; PEDROSA, C.E.; OLIVEIRA, C.M. de; DORNAS, M.F.S.; CRUZ, C.D.; VALADARES, N.R. Application of artificial neural networks in indirect selection: a case study on the breeding of lettuce. Bragantia, v.74, p.387-393, 2015. DOI: 10.1590/1678-4499.0088.

BERGMEIR, C.; BENÍTEZ, J.M. Neural networks in R using the Stuttgart Neural Network Simulator: RSNNS. Journal of Statistical Software, v.46, p.1-26, 2012. DOI: 10.18637/jss.v046. i07.

BLEINROTH, E.W. Matéria-prima. In: MEDINA, J.C.; BLEINROTH, E.W.; MARTIN, Z.J.; TRAVAGLINI, D.A.; OKADA, M.; QUAST, D.G.; HASHIZUME, T.; MORETTI, V.A.; BICUDO NETO, L.C.; ALMEIDA, L.A.S.B.; RENESTO, O.V. (Ed.). Banana: cultura, matéria-prima, processamento e aspectos econômicos. 2.ed. Campinas: ITAL, 1990. p.133-196.

COELHO, S.R.M.;WERNER, S.S.; PONCIO,A.P.;FERREIRA,L.; NÓBREGA, L.H.P. Performance during post-harvest storage of banana cv. 'Prata', 'Maçã' and 'Nanica' exposed to physical and chemical treatments. Engenharia Agrícola, v.31, p.727-734, 2011. DOI: $10.1590 / \mathrm{S} 0100-69162011000400011$

DAGAR, A.; WEKSLER, A.; FRIEDMAN, H.; LURIE, S. Gibberellic acid $\left(\mathrm{GA}_{3}\right)$ application at the end of pit ripening: effect on ripening and storage of two harvests of 'September Snow' peach. Scientia Horticulturae, v.140, p.125-130, 2012. DOI: 10.1016/j. scienta.2012.03.013.

FINGER, F.L.; PUSCHMANN, R.; BARROS, R.S. Effects of water loss on respiration, ethylene production and ripening of banana fruit. Revista Brasileira de Fisiologia Vegetal, v.7, p.115-118, 1995.

FOOD AND AGRICULTURE ORGANIZATION OF THE UNITED NATIONS. FAOSTAT. Disponível em: <http://faostat. fao.org/site/339/defaut.aspx>. Acesso em: 1 dez. 2015.

GARSON, G.D. Interpreting neural network connection weights. International Journal of Artificial Intelligence and Expert Systems, v.6, p.47-51, 1991.

GHORBANI, M.A.; KHATIBI, R.; GOEL, A.; FAZELIFARD, M.H.; AZANI, A. Modeling river discharge time series using support vector machine and artificial neural networks. Environmental Earth Sciences, v.75, p.685-698, 2016. DOI: 10.1007/s12665-016-5435-6

HUANG, H.; JING, G.; WANG, H.; DUAN, X.; QU, H.; JIANG, Y. The combined effects of phenylurea and gibberellins on quality maintenance and shelf life extension of banana fruit during storage. Scientia Horticulturae, v.167, p.36-42, 2014. DOI: 10.1016/j. scienta.2013.12.028

KHADEMI, F.; BEHFARNIA, K. Evaluation of concrete compressive strength using artificial neural network and multiple linear regression models. International Journal of Optimization in Civil Engineering, v.6, p.423-432, 2016.

LATTIN, J.; CARROLL, J.D.; GREEN, P.E. Análise de dados multivariados. São Paulo: Cengage Learning, 2011. 455p.

LICHTEMBERG, L.A.; LICHTEMBERG, P. dos S.F. Avanços na bananicultura brasileira. Revista Brasileira de Fruticultura, v.33, p.29-36, 2011. Número especial. DOI: 10.1590/ S0100-29452011000500005. 
MODESTO, J.C.; RODRIGUES, J.D.; ONO, E.O.; HABERMANN, G. Aplicação de ácido giberélico $\left(\mathrm{GA}_{3}\right)$ em pré-colheita de tangerina 'Poncã' (Citrus reticulata Blanco). Acta Scientiarum. Agronomy, v.28, p.3740, 2006. DOI: 10.4025/actasciagron.v28i1.1297.

MOHAPATRA, D.; MISHRA, S.; SINGH, C.B.; JAYAS, D.S. Post-harvest processing of banana: opportunities and challenges. Food Bioprocess Technology, v.4, p.327-339, 2010. DOI: 10.1007/s11947-010-0377-6.

NEWILAH, G.N.; DHUIQUE-MAYER, C.; ROJAS-GONZALEZ, J.; TOMEKPE, K.; FOKOU, E.; ETOA, F.X. Carotenoid contents during ripening of banana hybrids and cultivars grown in Cameroon. Fruits, v.64, p.197-206, 2009. DOI: 10.1051/fruits/2009015.

NGUYEN, T.B.T.; KETSA, S.; DOORN, W.G. van. Relationship between browning and the activities of polyphenoloxidase and phenylalanine ammonia lyase in banana peel during low temperature storage. Postharvest Biology and Technology, v.30, p.187-193, 2003. DOI: 10.1016/S0925-5214(03)00103-0.

PRILL, M.A. de S.; NEVES, L.C.; CHAGAS, E.A.; TOSIN, J.M.; SILVA, S.S. Métodos para a climatização de bananas 'Prata-Anã' produzidas na Amazônia setentrional brasileira. Revista Brasileira de Fruticultura, v.34, p.1030-1042, 2012. DOI: 10.1590/ S0100-29452012000400009.

R CORE TEAM. R: A language and environment for statistical computing. Vienna: R Foundation for Statistical Computing, Vienna, 2013.
ROSSETTO, M.R.M.; LAJOLO, F.M.; CORDENUNSI, B.R. Influência do ácido giberélico na degradação do amido durante o amadurecimento da banana. Ciência e Tecnologia de Alimentos, v.24, p.76-81, 2004. DOI: 10.1590/S0101-20612004000100015.

SMITH, J.H.C.; BENÍTEZ, A. Clorophylls: analysis in plant materials. In: PAECH K.; TRACEY M.V. (Ed.). Modern methods of plant analysis. Berlin: Spring-Verlag, 1955. v.4, p.142-196.

SOARES, F.C.; ROBAINA, A.D.; PEITER, M.X.; RUSSI, J.L.; VIVAN, G.A. Redes neurais artificiais na estimativa da retenção de água do solo. Ciência Rural, v.44, p.293-300, 2014. DOI: 10.1590/S0103-84782014000200016.

STOJANOVIC, B.; MILIVOJEVIC, M.; MILIVOJEVIC, N.; ANTONIJEVIC, D. A self-tuning system for dam behavior modeling based on evolving artificial neural networks. Advances in Engineering Software, v.97, p.85-95, 2016. DOI: 10.1016/j. advengsoft.2016.02.010.

WEKSLER, A.; DAGAR, A.; FRIEDMAN, H.; LURIE, S. The effect of gibberellin on firmness and storage potential of peaches and nectarines. Acta Horticulturae, v.962, p.591-595, 2012. DOI: 10.17660/ActaHortic.2012.962.80.

YANG, X.; PANG, X.; XU, L.; FANG, R.; HUANG, X.; GUAN, P.; LU, W.; ZHANG, Z. Accumulation of soluble sugars in peel at high temperature leads to stay-green ripe banana fruit. Journal of Experimental Botany, v.60, p.4051-4062, 2009. DOI: 10.1093/ jxb/erp238.

Recebido em 4 de janeiro de 2016 e aprovado em 5 de maio de 2016 